\title{
Review of Clinical and Functional Outcome of Prosthetic Salvage for Infected Total Knee Replacement
}

\section{Janipireddy SB*}

SBA Jariwala Tayside NHS Hospitals, Dundee, UK

\begin{abstract}
We have retrospectively studied the infected total knee replacement cases treated by open irrigation and debridement from 1996 to 2006 at NHS Tayside hospitals. Case notes of 18 (17 patients) were reviewed and looked into factors such as criteria chosen for surgery, timing of debridement, organism cultured and antibiotic duration. A success rate of $94 \%$ was noted (only 1 case of failure). Failure of the procedure included those which were re-infected or underwent some other treatment after initial debridement. The clinical and functional outcome amongst the successful cases was noted to be good as evidenced by knee society scores. The median follow-up was 3 years. The commonest organism found was staphylococcus aureus $(27 \%)$. The single case of failure was a 77 year old female with no significant co-morbidities and had mixed organisms in culture. No relation was found between success and failure with regard to age, sex, pre-operative diagnosis and co-morbidity. However, timing of surgery and the length of antibiotic administration were considered relevant to the success of this method. Despite the hesitant and not so encouraging results of some previous studies concerning prosthetic salvage by this method, we believe that this procedure has a role in immediate post-operative period in carefully chosen cases within 4 weeks of index surgery.
\end{abstract}

Keywords: Knee replacement; Patients; Hospital

\section{Introduction}

Totalkneereplacement(TKR)isoneofthemostfrequentlyperformed prosthetic joint surgeries throughout the world today. Infection is one such complication that is dreaded by surgeons. The treatment options in an infected TKR can be grouped as those with prosthetic retention and others involving their removal. The "gold standard" in treatment of infected TKR is a two-stage revision arthroplasty, which boasts an $80 \%$ success rate [1]. This procedure is time-consuming, expensive and also gives varying functional outcomes. The second option is prosthetic salvage. The role of knee debridement with prosthetic retention in infected cases is challenging. The success of such a procedure relies upon multiple criteria such as; timing of surgery in relation to primary TKR or presentation of symptoms, type of infection (early, late) and the type of organism present. Irrigation and debridement had good results if performed within 2 weeks of index surgery $[2,3]$. Results were not encouraging when performed in cases infected with staphylococcus and streptococcus $[4,5]$. These studies help us to recognise the fact that early diagnosis and prompt intervention could result in better outcome. Knee irrigation and debridement, if successful, would aid in a reduction in cost and also save a lot of waiting time for patients undergoing a twostage re-implantation. The aim of our study was to review the clinical and functional outcome of knee washouts. Additionally, factors such as age, co-morbidities and the criteria that made surgeons choose this particular procedure were looked into.

\section{Patients and Methods}

From 1996 to 2006, 29 cases (28 patients) were treated by irrigation and debridement at Tayside NHS Hospital (NHS: National Health Service UK), Scotland by a variety of surgeons. Only 18 cases (17 patients) could be included for this retrospective study. The reason for exclusion of the remaining cases was that, case records of 10 patients were missing and in 1 case there was no satisfactory documentation and further follow-up after 6 months. All the patients in the study group were operated by open irrigation and debridement. Caldicott approval was sought and the medical records of these patients were investigated.
The data were collected using a Pro forma sheet, which involved the variables shown in Table 1. Because of the small number of cases, it was appropriate to view each variable in terms of percentage and their frequency. It was difficult to demonstrate any correlation between a variable and the outcome (Table 1).

Variables such as Timing of surgery, organisms and co-morbidities were analysed if they had any relevance in terms of functional outcome. Descriptive statistics were derived using SPSS software programme (SPSS: Statistical Package for the Social Sciences/IBM). The final outcome of success of this procedure was based upon an infectionfree period of 2 years with no evidence of loosening on radiological evaluation [6]. Knee score, functional scores and blood parameters were recorded at each follow-up until the patient was declared free from infection by the consultant. Recurrence of infection, loosening viewed on X-ray or subsequent removal of the prosthesis was considered to be failure. Functional outcome of the successful cases were evaluated by recording the knee society scoring system [7] (Table 2).

\begin{tabular}{|l|l|l|l|}
\hline Continuous & Discrete & Categorical & Nominal \\
\hline (1) Age & & (1) CRP & (1) Sex \\
(2) Knee scores & (2) WBC count & (2) Organisms \\
(3)Time since index surgery & of follow-up & (3) Skin condition & (3) Co-morbidities \\
(4) Time of presentation & (4) X-ray & (4) Outcome \\
(5) Duration of antibiotic given & & (5) Special tests & \\
\hline
\end{tabular}

Table 1: Variables used for data collection.

*Corresponding author: Satish Babu Janipireddy, North Middlesex University Hospital London, Middlesex, United Kingdom, Tel: 0044-7895273757; E-mail: satishbabu74@gmail.com

Received March 20, 2017; Accepted April 11, 2017; Published April 18, 2017

Citation: Janipireddy SB (2017) Review of Clinical and Functional Outcome of Prosthetic Salvage for Infected Total Knee Replacement. Health Care Current Reviews 5: 192. doi: 10.4172/2375-4273.1000192

Copyright: ( 2017 Janipireddy SB. This is an open-access article distributed under the terms of the Creative Commons Attribution License, which permits unrestricted use, distribution, and reproduction in any medium, provided the original author and source are credited. 


\begin{tabular}{|l|l|}
\hline Knee society \\
\hline Knee society Rating \\
\hline Pain (50 points) \\
\hline None \\
\hline Mild or occasional \\
\hline Stains only \\
\hline Moderate continual & \\
\hline severe & \\
\hline Range of motions degrees & \\
\hline 1 point & \\
\hline
\end{tabular}

\begin{tabular}{|c|c|}
\hline Points & Patient score \\
\hline 50 & $=50$ \\
\hline 45 & \\
\hline 30 & \\
\hline 20 & \\
\hline 10 & \\
\hline 25 & $=25$ \\
\hline 0 & \\
\hline
\end{tabular}

Anteroposterrior stability (minimum movement in any position)

\begin{tabular}{|l|c|c|}
\hline$<5 \mathrm{~mm}$ & 10 & $=10$ \\
\hline $5-10 \mathrm{~mm}$ & 5 & \\
\hline $10 \mathrm{~mm}$ & 0 & \\
\hline
\end{tabular}

\section{Medial lateral stability}

\begin{tabular}{|c|c|c|}
\hline$<5$ degrees & 15 & $=15$ \\
\hline $6-9$ degrees & 10 & \\
\hline 10-14 degrees & 5 & \\
\hline 15 degrees & 0 & \\
\hline \multicolumn{3}{|l|}{ Deductions } \\
\hline Flexion contracture & & $=0$ \\
\hline 5-10 degrees & 2 & \\
\hline 10-15 degrees & 5 & \\
\hline 16-20 degrees & 10 & \\
\hline$>20$ degrees & 15 & \\
\hline \multicolumn{3}{|l|}{ Extension log } \\
\hline$<10$ degrees & 5 & \\
\hline 10-20 degrees & 10 & \\
\hline$>20$ degrees & 15 & \\
\hline \multicolumn{3}{|l|}{ Alignment } \\
\hline 5-10 degrees & 0 & \\
\hline $0-4$ degrees & 3 points each & \\
\hline 11-15 degrees & 3 points each & \\
\hline \multicolumn{3}{|l|}{ Other } \\
\hline \multicolumn{3}{|l|}{ Function rating } \\
\hline Walking & & $=50$ \\
\hline \multicolumn{3}{|l|}{ Unlimited } \\
\hline$>10$ blocks & 40 & \\
\hline $5-10$ blocks & 30 & \\
\hline$<5$ blocks & 20 & \\
\hline House bound & 10 & \\
\hline Unable & 0 & \\
\hline Stains & & $=50$ \\
\hline Normal up and down & 50 & \\
\hline Normal ups and down with rail & 40 & \\
\hline up and down with rail & 30 & \\
\hline up with rails unable down & 15 & \\
\hline unable & 0 & \\
\hline Deductions & & $=0$ \\
\hline Cane & 5 & \\
\hline Two canes & 10 & \\
\hline Walker & 20 & \\
\hline \multicolumn{3}{|l|}{ Score } \\
\hline Knee Rating & 100 & \\
\hline Function & 100 & \\
\hline \multicolumn{3}{|c|}{ (Adapted from=install JN, OCCR 1989;248:12) } \\
\hline
\end{tabular}

Table 2: Knee society scoring system.

\section{Results}

There was only 1 reported failure amongst the 18 cases in our study. All the patients were primarily operated by using cemented prosthesis. The patients were evaluated and found to be radio logically stable before the procedure. The different variables and their results are as below are described as below.

\section{Age, sex and diagnosis}

The study group consisted of 14 women (72\%) and 4 men (28\%). The pre-operative diagnosis was osteoarthritis in 14 knees, rheumatoid arthritis in 3 and psoriatic arthropathy in 1 . The age range at which the debridement was done was between 54 and 82 years (median age, 70 years). The single case, which was reported as failure, was a 77 year old female operated for osteoarthritis.

\section{Criteria for debridement}

There were no clear criteria recorded in the case notes as to why irrigation and debridement was chosen. However, data were collected for presentation of symptoms, laboratory investigations such as leukocyte count and CRP (C-reactive Protein) count. X-ray reports were recorded. There was no radioisotope study performed. The main symptoms and signs observed were occurrence of new pain, wound dehiscence and wound discharge. Wound dehiscence (10 cases, 55\%) was the most common presentation for which the patient was operated upon by this method. Seven patients $(39 \%)$ had wound discharge, either blood ooze or serous discharge. Only 1 patient (5\%) was recorded to have occurrence of new pain. White blood cell (WBC) count was the most common laboratory test documented in the case notes. WBC counts were documented in 17 of the total number of 18 cases. They were normal before and after the surgery in 10 cases (59\%). They were elevated in the remaining 7 cases. The WBC count was normal before and after the surgery in the single case where there was a failure. WBC count was raised pre-operatively in only 2 cases which later grew microorganisms on culture. A CRP count of over $20 \mathrm{mg} / \mathrm{L}$ was considered to be raised. CRP was recorded in only 7 cases. In other cases, there was no mention of CRP both in the pre-operative and post-operative period. It was grossly elevated (above 300) in 5 cases out of 7. CRP was also used as a post-operative tool for prognosis and for regulation of antibiotics.

\section{Co-morbidity}

The co-morbidities which were significant to the procedure were diabetes, rheumatoid and related conditions, previous surgeries around the knee, patients taking steroid therapy and immunosuppressive therapy and those diagnosed as malignancy of other systems. The co-morbidities observed in our study were as shown in Figure 1. There was no significant co-morbidity observed in the failure case at the time of the procedure. This patient was hypertensive, which was not considered as a significant comorbidity for this procedure. However, she had a history of intra-operative fracture of medial femoral condyle. She was subsequently followed-up and the X-rays showed gross collapse of the condyle.

\section{Micro-organisms}

Specimens that were sent for culture were mostly from wound swab taken per-operatively and post-operatively. There were no organisms found in $44 \%$ of cases. Amongst the positive culture groups, the most common organisms recorded were Staphylococcus aureus, methicillin resistant Staphylococcus aureus (MRSA), coagulase-negative staphylococcus organism (CNS) and others (mainly entero-cocci, pseudomonas and corynebacterium species). Five cases had cultured 

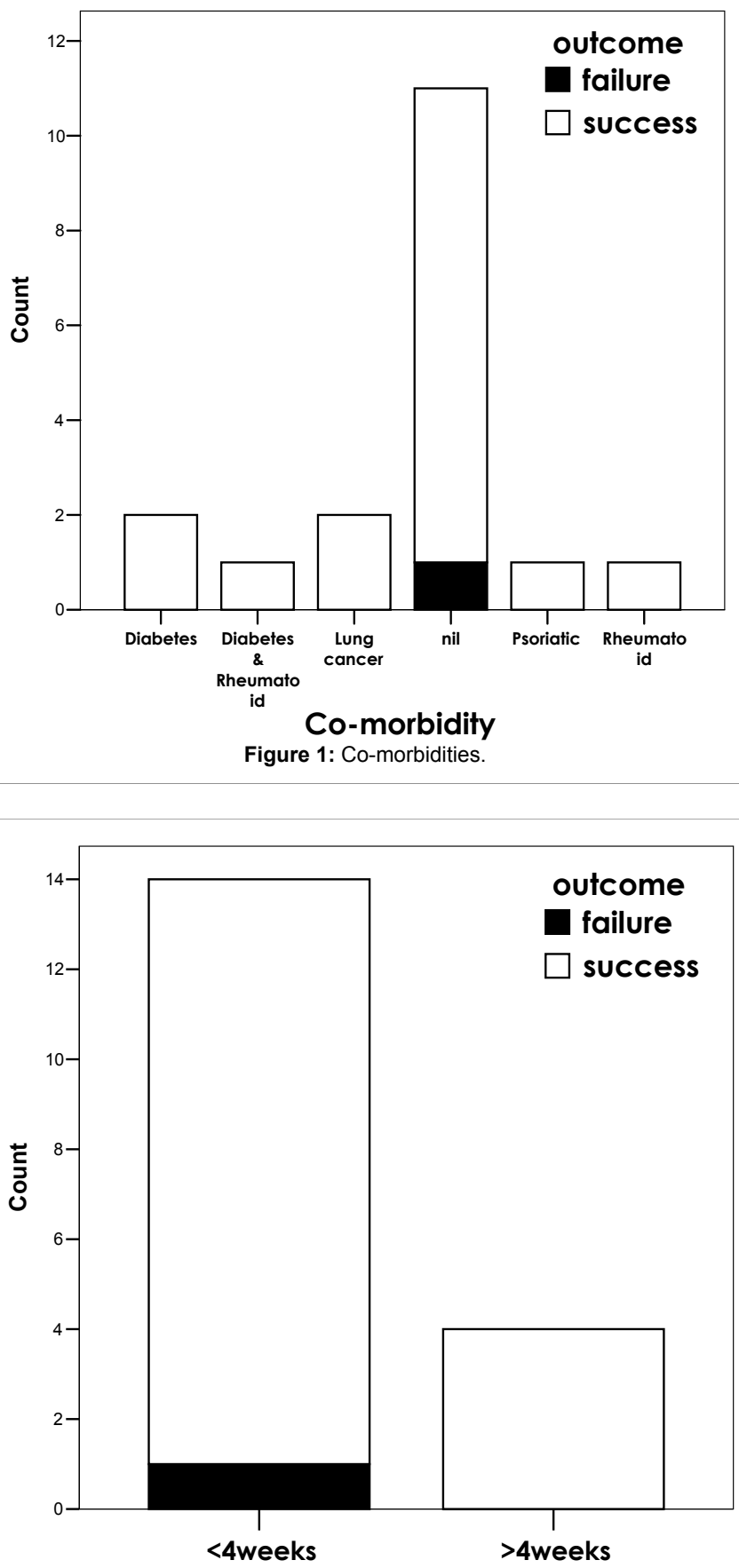

Figure 2: Timing since index surgery.

staphylococcus organisms (16\% were MRSA and $11 \%$ staph. aureus). Four cases (22\%) had CNS organism. There was 1 case (5\%) with mixed growth of organisms (proteus and corynebacterium species). CNS and pseudomonas organism on late cultures from knee tissue in the postoperative period were found in the single failure case.

\section{Timing of the surgery}

The timing of debridement from the index surgery was noted in weeks. The mean time of surgery was from 1 week to 7 weeks (mean 2.83). For the purpose of comparison of our practise with that of similar studies, timing of surgery was grouped into those which were operated in less than 4 weeks and those more than 4 weeks. The frequency of cases operated from index surgery as compared to their outcome is shown in Figure 2.

\section{Surgical procedure}

Irrigation and debridement were performed in all cases by an open method. Twelve cases $(67 \%)$ were operated by irrigation and simple debridement. Haematoma was the main finding in the majority of these cases intra-operatively. The other 6 cases (33\%) required replacement of the plastic insert along with debridement. The reasons for these replacements were not well documented in the case notes. Of these 6 cases, 4 cases showed positive cultures ( 2 cases showed staphylococcus aureus and the other 2 showed CNS). The failure case was operated by plastic exchange at $5^{\text {th }}$ post-operative period in addition to irrigation and debridement.

\section{Antibiotic duration}

Antibiotics were administered in 17 cases out of the total of 18 . In 3 cases, a regular intravenous antibiotic was given even after cultures were negative for around 3 days, which was the time taken for the culture results. In the remaining 14 cases, the range of time of antibiotic was from 6 weeks to 14 weeks (median 8 weeks). One case, which was a failure and operated at 3 months, received antibiotics for 24 weeks. Antibiotic was stopped by the surgeon on the basis of clinical symptoms and signs, supported by normal WBC counts and return of CRP to within normal levels in 7 cases.

\section{Outcome}

Seventeen cases (94\%) were successful following this procedure. Follow-up ranged from 1 to 7 years. The median time of follow-up was 3 years. Their functional outcome was found by measuring the knee score and functional score. There was only 1 case that was reported as failure, as the patient required a revision surgery after 3 months of irrigation and debridement of the infected joint. The statistics shown in the following sections is calculated for the 17 successful cases excluding the single failure case. Knee scores were collected in all but 1 case (failure case). There was no pre-operative score recorded in 2 cases. Amongst the 17 cases (success cases), the range of knee score at final follow-up was from 41-99 (mean, 86.35). Scores of 70 to 100 (fair to excellent) were recorded in 15 cases $(88 \%)$ at final follow-up. The improvement in knee scores and function score are as shown in Figures 3 and 4 respectively. The details of 2 cases, which showed no improvement, were as follows: the first case was a male of 74 years of age with lung cancer and was operated at 6 weeks time. No organism was cultured, but the patient did receive antibiotics for a 10 week period post-operatively. This patient had a knee flexion of 105 degrees and a pain score of ' 1 ' at final follow-up. This

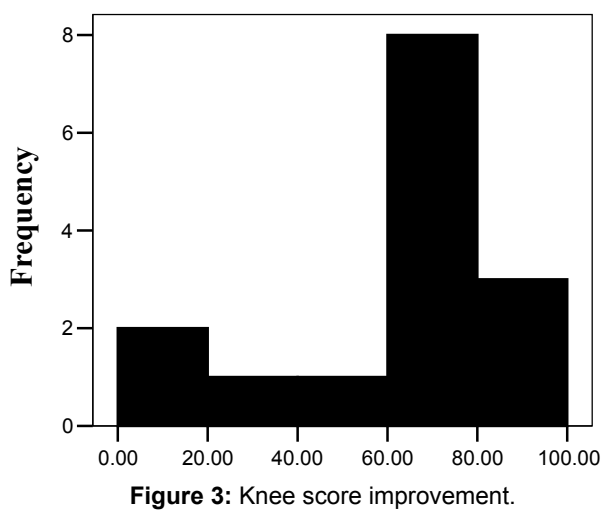




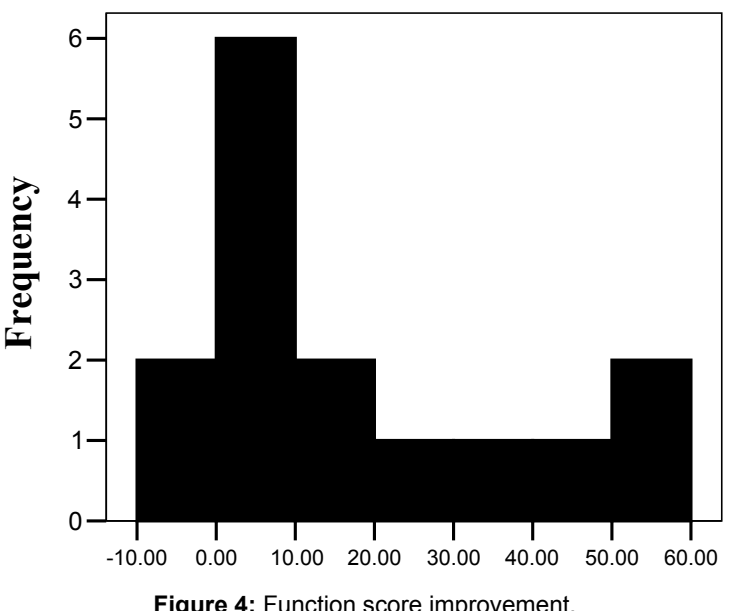

patient also had severe osteoarthritis of the opposite knee, which was due for a replacement. The second case was a 56 year old male, with no comorbidities who was operated at the end of 3 weeks. MRSA was cultured on 3 different occasions. This patient had a knee flexion of 100 degrees with ' 0 ' pain score. Both pre- and post-operative functional scores were recorded. Data could only be collected in 15 cases. Their scores ranged from 45-100 at final follow-up (mean, 62.8) (Figures 3 and 4) shows the Knee Score improvement and Functional score improvement (calculated as a difference between the pre operative and post operative at final follow-up). There were 2 cases, which showed deterioration of ' -10 ' in functional score and 5 cases showed no improvement in their scores compared to the pre-operative period. The first case, where deterioration was observed, was that of an 80 year old female patient, operated at 2 weeks time. There was no organism cultured and antibiotic was administered for a period of 1 week. This patient had a flexion range of 125 degrees and a pain score of ' 0 '. The other case was a 65 -year old female patient who was on treatment for psoriasis. She grew MRSA, was operated at 2 weeks time and received antibiotic for a period of 14 weeks post-operatively. This patient had a knee flexion of 100 degrees and a pain score of ' 0 '. Both these cases had poor general health secondary to multiple co morbidities. The patients with no improvement had good pre-operative functional scores anyway.

\section{Discussion}

The role of irrigation and debridement in the treatment of infected TKR is a debatable issue. This review of practise of prosthesis salvage in infected primary knee replacements at NHS Tayside Hospitals revealed that this was a successful procedure in all except 1 case. There were also a few studies which were operated by arthroscopic methods and felt that this procedure could be considered as an alternative to two-stage revision surgery in select cases [8-10]. This implies that prosthesis salvage is being tried in various centres worldwide. The aim of this current study was to investigate the case notes of patients who were operated by this method and discover important aspects of the notes, which led to the success of this procedure in terms of functional outcome. Most previous studies stressed the importance of the timing of the procedure in relation to the index surgery or the duration of symptoms [2,3,11]. Borden et al. [2] had good results in cases operated within 2 weeks of the index surgery. There were only 6 cases which were operated by this time, 5 of which were successful. In 1990 Schoifet and Morrey showed good results in cases which were operated within 4 weeks of the duration of symptoms. Their average duration was 21 days in which prosthesis was salvaged and 36 days in which the treatment was considered to be a failure. Trampuz et al. [1] had $96 \%$ success at one year, $92 \%$ at two years and $86 \%$ at three years. Our results are consistent with these authors showing a success rate of $94 \%$ at three years. However, no comment could be made for the failure case with regard to timing as it was operated in less than 1 week from index surgery. However, as the majority of cases were operated within 4 weeks, it can be stressed that this was a reasonably successful time limit for this procedure.

The commonest organism noted in prosthetic knee infections was staphylococcus aureus in the report by NINSS [12]. Our series had 5 cases $(27 \%)$ infected with staphylococcus organism (16\% were MRSA, $11 \%$ staphylococcus aureus). The next most common organism cultured was CNS (4 cases, 22\%). Diermengian et al. [4] reported $87 \%$ failure with staphylococcus organism and $45 \%$ with CNS and Schoifet and Morrey reported a 58\% failure with staphylococcus [11]. Our series showed complete success with staphylococcus species and only $25 \%$ failure amongst the CNS organism. The single case of failure with CNS organism could be attributed to it being a mixed growth along with pseudomonas. The most common presentation in our study was wound dehiscence (55\%). Other forms of presentation were wound discharge and occurrence of new pain. Previous studies described the type of presentation for inclusion into the study group but no clear association was found to any. In our series, wound discharge was noted in the failure case. Routine X-rays of the knee were done before the debridement procedure to make sure that the prosthesis was stable. No special tests (nuclear medicine) were performed. Blood tests were not considered reliable for surgical intervention, as even the CRP, which was significantly abnormal in the operated cases, was mainly done on the day of surgery. Spangehl's, criteria of debridement was that the organism be sensitive to antibiotic and that the prosthesis be stable [13]. Our study also had the same criteria chosen for selection of this procedure.

The clinical and functional outcome of the successful cases (based on criteria of no re-operation at 2 years period) was very good. Amongst the successful cases there was satisfactory clinical and functional result as evidenced from the knee society scoring system. There was no reduction in knee scores post-operatively in any case. There was also no correlation noted to the co-morbidity and the functional outcome in these cases. Likewise, there was no relation found to the type of debridement (with or without plastic exchange) carried out. Antibiotic was administered post-operatively by both intravenous and oral route based upon the culture sensitivity results. They were given for a median period of 8 weeks. This is much lesser than what the previous authors mentioned $[8,10]$. No relation was found between success and failure with regard to age, sex, pre-operative diagnosis and co-morbidity. It was extremely difficult to note the timing of surgery from the presentation of symptoms as documentation was not very supportive for this. These satisfactory results which were achieved following this procedure could be attributed to early intervention and duration of accurate antibiotics given post-operatively. The single case of failure was a 77 year old lady who presented with wound discharge on the $5^{\text {th }}$ postoperative day. This patient had a fracture of medial femoral condyle preoperatively with no significant co-morbidities. She grew both CNS and pseudomonas organisms from her wound site and tissue. She was operated by two stage revision surgery and hence recorded as a failure. Despite the hesitant and not so encouraging results of some previous studies concerning prosthetic salvage by this method, we believe that this procedure has a role in immediate post-operative period in carefully chosen cases within 4 weeks of index surgery. This is however with varying clinical and functional outcomes but with a successful salvage of the implants. 
Citation: Janipireddy SB (2017) Review of Clinical and Functional Outcome of Prosthetic Salvage for Infected Total Knee Replacement. Health Care Current Reviews 5: 192. doi: 10.4172/2375-4273.1000192

Page 5 of 5

The limitations of this study are that, it was conducted retrospectively, implying that the selection of patients into this group was biased. Only 18 cases $(64 \%)$ of the 28 in total could be included in this study. We did not use any statistical treatment due to small numbers for the study used. Also the treatment method carried out was by a variety of surgeons, thus lacking the homogeneity.

\section{References}

1. TrampuzA, Zimmerli W (2006) Diagnosis and treatment of infections associated with fracture-fixation devices. Int J Care injured 37: S59-S66.

2. Borden LS, Gearen PF (1887) Infected total knee arthroplasty: A protocol for management. J Arthroplasty 2: 27.

3. Teeny SM, Dorr L, Murata G, Conaty P (1990) Treatment of infected total knee arthroplasty. J Arthroplasty 5: 135.

4. Deirmengian C, Greenbaum J, Stern J, Braffman M, Lotke P A, et al. (2003) Open debridement of acute gram-positive infection after total knee arthroplasty. Clin Orthop Relat Res 416: 129-134.

5. Meehan AM, Osmon DR, Duffy MCT, Hansenn AD, Keating MR (2003) Outcome of pencillin-susceptible streptococcal prosthetic joint infection treated with debridement and retention of prosthesis. Clin Infect Dis 36: 845-849.
6. Hartman MB, Fehring TK, Jordan L, Norton HJ (1991) Periprosthetic knee sepsis. Clin Orthop Relat Res 273: 113-118.

7. Insall JN, Dorr LD, Scott RD, Scott WN (1989) Rationale of the Knee society clinical rating system. Clin Orthop 248: 13-14.

8. Dixon P, Parish EN, Cross MJ (2004) Arthroscopic debridement in the treatment of infected total knee replacement. J Bone Joint Surg (Br) 86: 39-42.

9. Waldman BJ, Hostin E, Mont MA, Hungerford DS (2000) Infected total knee arthroplasty treated by arthroscopic irrigation and debridement. J Arthroplasty 15: $430-436$.

10. Agarwal S (2004) Arthroscopic debridement in the treatment of infected knee replacement. J Bone Joint Surg Br 86: 1221.

11. Schoifet SD, Morrey BF (1990) Treatment of infection after Total knee arthroplasty by debridement with retention of the components. J Bone Joint Surg Am 72: 1383-1390.

12. Nosocomial infection national surveillance service (NINSS) (1997) Surveillance of surgical site infections in English hospitals.

13. SpangehI MJ, Masri BA, O'Connell JX, Duncan CP (1999) Prospective analysis of preoperative and intraoperative investigations for the diagnosis of infection at the sites of two hundred and two revision total hip arthroplasties. J Bone Joint Surg Am 81: 672-683. 